\title{
Concept Level Web Search Via Semantic Clustering
}

\author{
Nian Yan and Deepak Khazanchi \\ College of Information Science and Technology, \\ University of Nebraska at Omaha, NE 68182, USA \\ \{nyan, khazanchi\}@mail.unomaha.edu
}

\begin{abstract}
Internet search engine techniques have evolved from simple web searching using categorization (e.g., Yahoo) to advanced page ranking algorithms (e.g., Google). However, the challenge for the next generation of search algorithms is not the quantity of search results, but identifying the most relevant pages based on a semantic understanding of user requirements. This notion of relevance is closely tied to the semantics associated with the term being searched. The ideal situation would be to represent results in an intuitive way that allows the user to view their search results in terms of concepts related to their search word or phrase rather than a list of ranked web pages. In this paper, we propose a semantic clustering approach that can be used to build a conceptual search engine.
\end{abstract}

Keywords: Conceptual Search, Search Engine, Document Clustering, Information Retrieval.

\section{Introduction}

Internet search engine techniques have evolved from simple web searching using categorization (e.g., Yahoo) to advanced page ranking algorithms (e.g., Google) [1]. However, one critical disadvantage of this approach is that ranking related information is not semantically grouped. That is, the results are not organized by concepts but by rank. For example, if one enters the term "Java" in Google, the search engine does not know what exactly this word means - is it "coffee Java", "Java programming" or even "island Java". Indeed, all three of these different results will show up in the search results. An added problem is that the phrase "Java programming" is so popular that it will dominate at least the first ten pages of the search results. Another related issue in traditional search engines is the use of Boolean operation. Internet users retrieve information from massively indexed data by inputting keywords followed by Boolean operations such as AND, OR and NOT as the representatives of the query. The major disadvantage of Boolean operations is that the search results have less relevance compared with what the user is actually looking for [2][3]. For example, a query for "Information System" returns results with key words "Information", "System" and "Information System", regardless of the associated meaning of the phrase. Besides Boolean operations, keyword searching has two major problems caused by synonymy and polysemy (multiple expressions of one thing or the same expression with multiple meanings). 
The use of a "conceptual search engine" can provide clarity to users while accommodating the semantic content (and context) of a term or phrase being searched. The "concept" in a conceptual search is defined as "one overarching idea or topic present in a web page" [2]. An even broader view of conceptual search is to do what one does naturally in conversations with others. In human conversations, we are able to understand the meaning of specific text or media, including web pages, plain text, and any documents consist of text message. This characteristic should be mirrored in a conceptual search engine. Conceptual searching will look for patterns of relationships rather than for individual words. We are in some sense focused on the idea of understanding the "text" rather than just querying for the "text".

We define a "concept" as the abstract of one stream of topics that users are interested in. The results of "conceptual searching" should be to display relevant contents that are organized by meaningful concepts. Thus, in this paper we explore the possibility of developing a conceptual search engine using a semantic clustering approach.

\section{Conceptual Searching}

There are two general approaches that could be used to achieve the goal of conceptual searching. One is to index the documents obtained from the internet by keywords and concepts based on an ontology system. In [4], a goal-oriented search engine was proposed to achieve an improvement compared with Boolean search. An adaptive search engine was developed that uses natural language processing to parse a user's search goal and uses "common sense" reasoning to translate the user's goal into an effective query. In this project, an Open Mind knowledge base (obtained from network contributors) is regarded as the recourse of "common sense". Another conceptual search engine implementation retrieves documents based on a combination of keyword and conceptual matching [5]. The documents are indexed automatically using the Open Directory ontology (Open Directory Project, http://dmoz.org), a distributed database of web content classified by internet volunteers. This approach for information retrieval of internet documents requires the support of an ontology system. It is unrealistic to build a conceptual search engine based on an ontology system maintained by volunteers because a well defined global version of an ontology system has not been achievable. Moreover, the general purpose of constructing ontology system is only for specific domain knowledge, e.g. an ontology system for the field of health informatics. In most cases, internet users are interested in one specific domain rather than seeking for information within a global knowledge domain.

An alternative to the previous ontology based approaches is to build conceptual search engines that can take internet documents and self-organized them into individual concepts. This can be used to return highly relevant concepts based on users' requests.

\section{Conceptual Searching Using Semantic Clustering}

In this section we describe our proposed solution as an alternative to the algorithms used in classical search engines by improving upon Boolean search and incorporating 
semantic clustering. In our solution, we assume that unstructured text data is preprocessed and then clustered. Fig. 1 illustrates the process we used in this paper to develop our proposed solution.

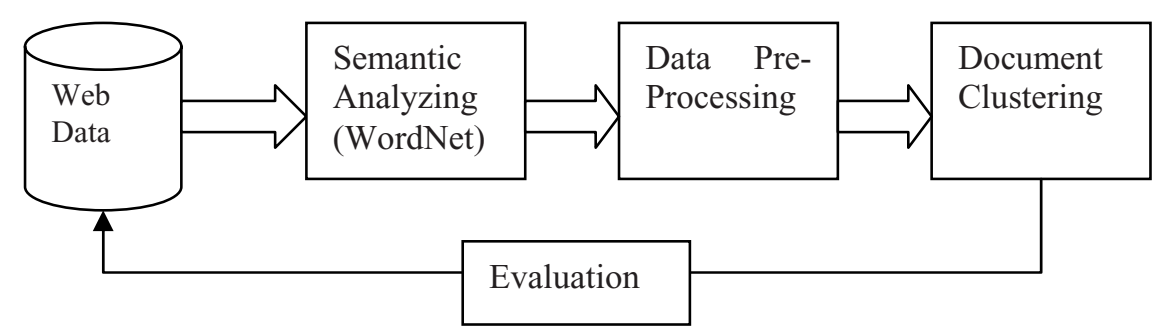

Fig. 1. Conceptual Searching by Semantic Clustering

\subsection{Data Preprocessing}

In order to analyze unstructured text data, preprocessing is implemented in three steps [6]: (1) Tokenization: dividing the documents into a set of index terms or keywords. (2) Stop-words: eliminating the most frequently used common English words, such as articles, prepositions, and conjunctions. (3) Stemming: prefixes and suffixes of the words are removed to reduce the redundancy of the same word in different phases. For example, "do", "doing", and "done" will be regarded as one word after stemming [7]. The documents are then processed by Vector Space Model [8] into a matrix with rows and columns representing documents and words respectively.

\subsection{Data Sets}

\section{Google Search Data Set}

This data source is directly collected from the internet. It aims to refine the search results of Google. Given a search term or phrase, Google's algorithm returns search results containing those key words ranked by a relevance score. It is necessary to design a query optimizer to extend the user's query to the range of key words derived from a thesaurus or even better lexical reference system, e.g. WordNet (http://wordnet.princeton.edu/). In this paper, the WordNet is used for semantically analyzing the user's input. The query optimizer then extends the original query, e.g. from term "Java" to "Java programming", "Java coffee" and "Java island" by referencing the thesaurus. We collected five cases from search results for each extended query. We use Google search API (http://code.google.com/apis/ajaxsearch/) to program and save the results.

\section{UCI KDD Biomedical Data Set}

This data set is part of the Syskill and Webert's web pages ranking data at UCI Machine Learning Repository (http://www.ics.uci.edu/ mlearn/MLRepository.html). We choose this dataset because many biomedical research applications used document clustering in, e.g. [6]. 


\subsection{Semantic Clustering}

In [9], a concept-driven search engine is designed and implemented (Carrot2, http:// www.carrot2.org) based on clustering search results from traditional search engines, e,g, Google, Yahoo and etc. The search results from traditional search engines are preprocessed using Vector Space Model and latent semantic indexing technique and then clustered [9].

Document clustering varies in performance under different problem settings [10]. Certainly, developing high-performance clustering algorithms could improve document clustering. On the other hand, it is important to develop a good document modeling method for improving the performance of document clustering. In some situations, the lack of class-specific "core" words leads to the failure of correctly clustering the documents. In [11], the authors proposed a semantic smoothing method for document models for the process of clustering. A proper set of core words are selected semantically. As a result, for each document, general words can be discounted. We believe that document modeling plays a more important role compared with other aspects of document clustering. A semantic document model is proposed based on the Vector Space Model. Different from [11], we develop the core word list as illustrated in the algorithm in Fig. 2. We use k-means, the classical partitional clustering algorithm, to cluster the documents. However, the number of $k$ in $\mathrm{k}$-means is not easy to determine. We have to experiment within a range from $k=$ 2 , and $k<8$. The reason $k$ stops at seven is because of the inherent limitation of human beings to process no more than seven different concepts at the same time. Any more concepts will result in information overload and confusion in the mind of the user [12].

\section{Step 1: Tokenizing}

Step 2: Eliminating the stop words

Step 4: Stemming then generate word list $L=\left\{x_{1}, \ldots, x_{n}\right\}$, porter stemming is used.

Step 5: Semantically Analyzing user's queries by WordNET, and generating initial core words list $C=\left\{t_{0}, \ldots, t_{n}\right\}$

Step 6: Adding more core words by analyzing the text sources, if $t_{i} \subset\left\{x_{j} \mid f_{\text {low }}<t f_{x_{j}}<f_{\text {high }}, j=1, \ldots, n\right\},(i=1, \ldots, m)$, where $t f$ denotes term frequency and $f_{\text {low }}, f_{\text {high }}$ denotes the threshold of the word frequency which is regarded significant to the final core word list. Those two parameters are determined by the experienced threshold or analyze of the human experts.

Thus, the core word list is: $C=\left\{t_{0}, \ldots, t_{n}, t_{n+1}, \ldots, t_{m}\right\}$

Step 7: Applying Vector Space model (TF/IDF)

Step 8: Clustering the documents by k-means, $\mathrm{k}=2, \ldots, 7$

Step 9: Evaluating the clusters by inter-distance. The longer the inter-distance is, the better the cluster will perform

Step 10: Presenting each cluster as an individual concept

Fig. 2. Semantic Clustering based on Vector Space Model 


\subsection{Simulations}

The simulations are implemented in Java and the WVTools Java package (http://rapid-i.com/content/view/21/44/lang,en/) is used for data preprocessing.

\section{Simulation 1: Clustering Google's Search Results}

In this part, we design a pilot study on refining the Google's search into conceptual level. That is, using semantic cluster to identify the major streams in terms of concepts. For this case, we already know $k=3$ because there are three different topics and for each one we collect five cases. We apply the algorithm in Fig. 2, clustering results are shown in Table 1 . There is only one web page that belongs to cluster 3 but is clustered into cluster 2 . Thus, the accuracy rate is $93.3 \%$.

Table 1. Number of Cases in each Cluster

\begin{tabular}{|c|c|c|}
\hline Cluster & Cases & Incorrectly clustered \\
\hline 1 & 5 & 0 \\
\hline 2 & 6 & 1 \\
\hline 3 & 4 & 0 \\
\hline Total Cases & 15 & \\
\hline
\end{tabular}

\section{Simulation 2: Conceptual Search for UCI Biomedical Data Set}

We used this data set to apply semantic clustering to identify the major streams in terms of concepts. In this case, after the step of stemming, the term frequency of each word is shown in Fig. 3. By a further human inspecting, we set $f_{\text {low }}=2, f_{\text {high }}=42$ and do the preprocessing again to get new core word list, after that there are 2051 words generated. By comparing the mean of inter-distance of the clusters under the different $k$ (shown in Fig. 4), we found that when $k=2$, the clustering performs best.

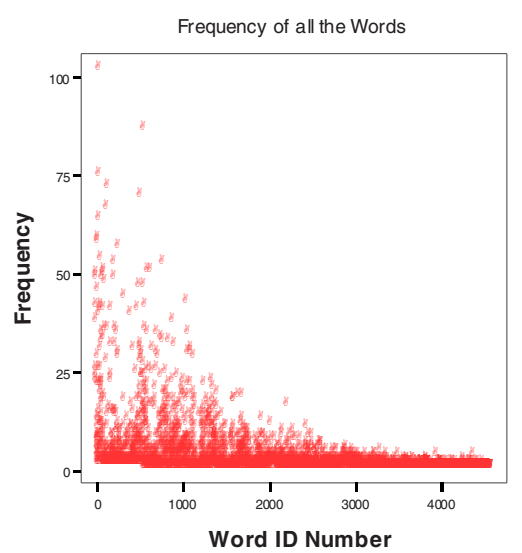

Fig. 3. Term Frequency

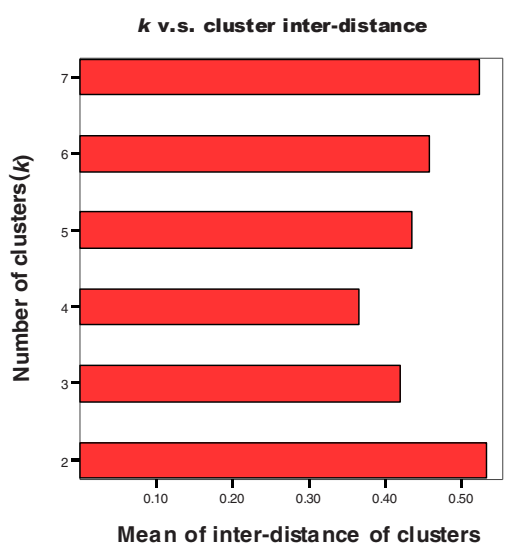

Fig. 4. Mean of inter-distance 
When $\mathrm{k}=2$, the two clusters have 4 and 132 pages, respectively. The contents of the clusters shows the fours are materials definitely not related to biomedical, e.g. networking, or invalid web pages. Thus we recommend $k=7$, when the cluster performs little worse than $k=2$ but more reasonable. The four cases are still in one cluster as well as when $k=2$. The other six clusters are the representatives of the different topic streams. This result is shown in Table 2.

Table 2. Number of Cases in each Cluster

\begin{tabular}{|c|c|c|c|c|c|c|c|c|}
\hline Cluster & 1 & $\mathbf{2}$ & 3 & 4 & 5 & 6 & $\mathbf{7}$ & Total Cases \\
\hline Cases & 46 & 18 & 4 & 11 & 13 & 30 & 14 & 136 \\
\hline
\end{tabular}

\section{Conclusions}

In this paper, we developed a practical approach for implementing conceptual searching on the internet. The web documents obtained from a standard search are semantically modeled into vectors for clustering analysis. The simulation results show the effectiveness of this approach. For example, in simulation 1, the accuracy of correctly assigning a document to a given conceptual cluster is $93.3 \%$. In this research, the identification of the topic for each cluster as the name of the concepts remains a good research topic in the future. For example, automatically creating a summary of the essential semantic content of a single web page for real time analysis without any other pre-processing, indexing and storing of the data from web needs to be further researched [2] [13]. Conceptual clustering techniques, such as COBWEB [14], contribute to creating the descriptions for clusters by incrementally creating hierarchical structure of the documents. Finally, the possible improvements that can be made in future research for this application is to analyze the document in phrases other than individual words (tokenization). Thus for example, the recognition of "software process improvement" as a phrase makes more sense for the Vector Space Model as compared with tokenization. The POS-tag [15] is a phrasing technique that can be used to make more sense of the data during pre-processing.

\section{References}

1. Page, L., Brin, S., Motwani, R., Winograd, T., The PageRank Citation Ranking: Bringing Order to the Web, Stanford University (1999)

2. Ramirez, P. and Mattmann, C., ACE: Improving Search Engines via Automatic Concept Extraction. Proceedings of the 2004 IEEE International Conference on Information Reuse and Integration (IEEE-IRI 2004), pp. 229-234. Las Vegas, Nevada, USA, November 8th$10^{\text {th }}(2004)$

3. Deniston, M., An Overview and Discussion of Concept Search Models and Technologies, Concept Searching Whitepaper, Fios Confidential (2003)

4. Liu, H., Lieberman, H., Selker, T., GOOSE: A Goal-Oriented Search Engine with Commonsense, Proceedings of the Second International Conference on Adaptive Hypermedia and Adaptive Web-Based Systems, pp. 253-263 (2002) 
5. Gauch, S., Madrid, J., Induri, S., Ravindran, D. and Chadlavada, S., KeyConcept: A Conceptual Search Engine, Information and Telecommunication Technology Center, Technical Report: ITTC-FY2004-TR-8646-37, University of Kansas (2004)

6. Peng, Y., Yan, N., Kou, G., Chen, Z., Shi, Y., "Document Clustering in Antimicrobial Peptides Research", Proceedings of the Eleventh Americas Conference on Information Systems, August 11-14 (2005)

7. Baeza-Yates, R. and Ribeiro-Neto, B. Modern Information Retrieval. Addison-Wesley, Wokingham, UK (1999)

8. Porter, M.F., An algorithm for suffix stripping, Program, 14(3): 130-137 (1980)

9. Osinski, S, and Weiss, D., A Concept-Driven Algorithm for Clustering Search Results, IEEE Intelligent Systems, vol. 20, no. 3, pp. 48-54, May/June (2005)

10. Zamir, O., Etzioni, O., Web document clustering: A feasibility demonstration. Proceedings of the 21st Annual International ACM Conference on Research and Development in Information Retrieval (SIGIR), 46-54 12 (1998)

11. Zhou, X., Zhang, X., and Hu, X., Semantic Smoothing of Document Models for Agglomerative Clustering, accepted in the Twentieth International Joint Conference on Artificial Intelligence (IJCAI 2007), Jan. 6-12, India (2007)

12. Miller, G. A., The Magical Number Seven, Plus or Minus Two: Some Limits on Our Capacity for Processing Information, The Psychological Review, vol. 63, pp. 81-97 (1956)

13. Michalski, R. S., "Knowledge acquisition through conceptual clustering: A theoretical framework and an algorithm for partitioning data into conjunctive concepts". International Journal of Policy Analysis and Information Systems 4: 219-244 (1980)

14. Fisher, D., "Knowledge acquisition via incremental conceptual clustering," Machine Learning, vol. 2, no. 2, pp. 139-172 (1987)

15. Hage, W., Rijke, M., and Marx, M., Information retrieval support for ontology construction and use, ISWC 2004: international semantic web conference, Hiroshima, Japan (2004) 